University of Nebraska - Lincoln

DigitalCommons@University of Nebraska - Lincoln

\title{
Survivable Waveband Switching in WDM Mesh Networks under Dedicated Path-Protection
}

\author{
Mengke Li \\ University of Nebraska-Lincoln, mli@cse.unl.edu \\ Byrav Ramamurthy \\ University of Nebraska-Lincoln, bramamurthy2@unl.edu
}

Follow this and additional works at: https://digitalcommons.unl.edu/cseconfwork

Part of the Computer Sciences Commons

Li, Mengke and Ramamurthy, Byrav, "Survivable Waveband Switching in WDM Mesh Networks under Dedicated Path-Protection" (2005). CSE Conference and Workshop Papers. 72.

https://digitalcommons.unl.edu/cseconfwork/72

This Article is brought to you for free and open access by the Computer Science and Engineering, Department of at DigitalCommons@University of Nebraska - Lincoln. It has been accepted for inclusion in CSE Conference and Workshop Papers by an authorized administrator of DigitalCommons@University of Nebraska - Lincoln. 


\title{
Survivable Waveband Switching in WDM Mesh Networks under Dedicated Path-Protection
}

\author{
Mengke Li and Byrav Ramamurthy \\ Department of Computer Science and Engineering, \\ University of Nebraska-Lincoln, USA. \\ Email: $\{$ mli, byrav $\}$ cse.unl.edu \\ Tel: (402)472-1779, Fax: (402)472-7767
}

\begin{abstract}
This paper considers the problem of dedicated path-protection in wavelength-division multiplexed (WDM) mesh networks with waveband switching functionality under shared risk link group (SRLG) constraints. Two dedicated pathprotection schemes are proposed, namely the PBABL scheme and the MPABWL scheme. The PBABL scheme protects each working waveband-path through a backup waveband-path. The MPABWL scheme protects each working waveband-path by either a backup waveband-path or multiple backup lightpaths. Heuristic algorithms adopting random optimization technique are proposed for both the schemes. The performance of the two protection schemes is studied and compared. Simulation results show that both the heuristics can obtain optimum solutions and the MPABWL scheme leads to less switching and transmission costs than the PBABL scheme.
\end{abstract}

\section{INTRODUCTION}

Wavelength division multiplexing (WDM) is an important technique to provide high-speed data transmission. To reduce the provisioning costs in the optical domain of a WDM network, waveband switching (WBS) was introduced [1] to group a specific set of wavelengths into a waveband at an optical crossconnect (OXC). The waveband transmitted along some common links forms a waveband-path. An OXC with the functionality of WBS is called a multi-granular optical crossconnect (MG-OXC), which can switch connection requests at different granularities. This paper considers a two-layer MGOXC as shown in [2], including a wavelength-crossconnect (WXC) layer and a waveband-crossconnect (BXC) layer. In such an MG-OXC, wavelengths/wavebands are terminated or switched transparently through the WXC/BXC layer separately. The terminated waveband is demultiplexed into wavelengths, which are sent to the WXC layer as inputs. The output wavelengths at the WXC layer can be multiplexed selectively into a waveband, which is sent to the BXC layer as an input. The output wavelengths from the WXC layer and the output wavebands from the BXC layer are grouped and transmitted along the output fiber link.

In a WBS network, a component failure can cause the failures of several waveband-paths and lightpaths, leading to the loss of large amounts of data and revenue. Thus, fault management in WBS networks is of paramount importance. However, survivable WBS (SWBS) is a relatively unexplored topic, only gaining attention recently. The only related studies are [3] and [4], which address the problem without considering the routing problem or the shared risk link group (SRLG) constraint. To the best of our knowledge, the study of the SWBS problem including the routing problem and the SRLG constraint has never been carried out before.

To survive from a single network failure, dedicated pathprotection technique is adopted here in a WBS network under the SRLG constraints. Although dedicated path-protection [5] [6] [7] may not be the most efficient technique, it guarantees the network to be able to survive from any single point of failure. Thus, the choice of the protection scheme in solving the SWBS problem is reasonable. Dedicated path-protection in a WBS network is different from the one in a traditional WDM network [8] because it protects both the working lightpaths and the working waveband-paths. In addition, each link in the WBS network could belong to a failure risk group as addressed in [7]. An SRLG is a group of links with a shared vulnerability, such as a shared fiber cable or a shared right-of-way (ROW). Here, an SRLG risk represents a general risk. For the network to be survivable under any single point of failure, a backup path must be SRLG-diverse from its working path.

Two dedicated path-protection schemes are proposed, namely the Protecting-waveBand-At-waveBand-Level-only (PBABL) and the Mixed-Protection-At-waveBand-andWavelength-Level (MPABWL). Different objectives are considered for networks under different traffic loads. A network under light traffic load has no blocked traffic, where the goal is to minimize the operational costs in terms of utilized waveband/wavelength links. In a network under heavy traffic load, some traffic requests may be blocked because of the limited resources. The ultimate objective is to gain as much revenue as possible in such a network.

\section{Problem Statement And Protection Schemes}

\section{A. Network model}

The network consists of the MG-OXC nodes, each of which has the same fiber capacity and waveband capacity. The nodes are interconnected by optical fiber links with $W$ wavelengths and $B$ wavebands. The policy control plane of an MG-OXC selects at most $\theta=\left\lfloor\frac{W}{B}\right\rfloor$ specific wavelengths to be grouped into a waveband. $\theta$ is also referred to as waveband granularity. We do not consider fiber switching and wavelength 
and waveband conversions. Thus, a lightpath/waveband-path is subject to the wavelength/waveband continuity constraint. The grouping of wavelengths in a waveband is restricted to those from the same source and destined to the same destination. Without loss of generality, the wavelengths in a waveband are assumed to be contiguous. Moreover, a fiber link may contain multiple active (used) wavebands and multiple (used) active wavelengths at the same time.

The network can be expressed as a directed graph $G(V, E, W, B, \theta, L, R)$, where $V$ is the vertex set, $E$ is the edge set, $W$ is the wavelength set, $B$ is the waveband set, $\theta$ is the waveband granularity, $L$ is the set of link length constraints, and $R$ is the SRLG risk sets for links. The SRLG risk set of a link contains the risks that the link is vulnerable to. Two links are SRLG-diverse if they have no common risks. The SRLG risk set of a path contains all the risks that the links along the path are vulnerable to. Two paths are SRLG-diverse if they have no common risks. A link is SRLG-diverse to a path if their risk sets have no common element.

The connection requests between each node pair in the network is given by the traffic matrix. A connection request has the following attributes: a) It starts from a source MGOXC node and terminates at a destination MG-OXC node; b) It requires a whole wavelength capacity; c) It constrains the maximum path length; d) It generates a revenue value. Different connection requests may have different revenue values. Each connection request is transmitted and protected in a lightpath or in a waveband-path. Given a WBS network and a set of connection requests with the above attributes, the objective of the revenue maximization (revMAX) problem is to maximize the total revenue generated and the objective of the cost minimization (cstMIN) problem is to minimize the total costs incurred by successfully provisioned connection requests. The cost is represented by the total number of occupied waveband-links and wavelength-links in provisioning the connection requests.

\section{B. Protecting-waveBand-At-waveBand-Level-only (PBABL)}

For the PBABL scheme, each connection request is assigned with a working and backup path which are SRLG-diverse. A wavelength is assigned to each of the paths. If the waveband grouping requirement is satisfied, the PBABL scheme tries to set up a working waveband-path and a backup waveband-path under the condition that there are common free wavebands along both paths. For example, Fig. 1(a) shows a 5 node network with 7 links, where each link represents two unidirectional fibers in opposite directions that belong to a single SRLG. Besides that, all fibers covered by a dashed circle are in the same SRLG. When two connection requests from 1 to 5 arrive as shown in Fig. 1(b), PBABL finds out two free wavebands along the SRLG-diverse routes (1-2-3-5) and (1$4-5)$. Thus, a working waveband-path along route (1-4-5) and a backup waveband-path along route (1-2-3-5) are set up.

A working waveband-path will not be set up if no backup waveband-path is available. For example, in Fig. 1(c), no waveband-path is set up for another two connection requests

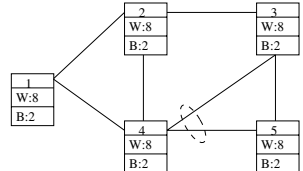

(a) Network Topology (W=8,B=2, $\theta=4)$

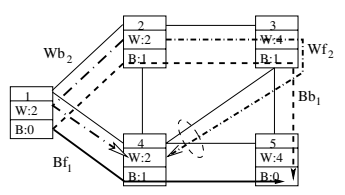

(c) PBABL with another 2 request from 2 to 4 $\longrightarrow$ Fiber Link $\longrightarrow$ Bf Working waveband-path $--->$ Bb Backup waveband-path

$-\rightarrow \rightarrow$ Wf Working lightpath $\quad-.-. \Rightarrow$ Wb Backup Lightpath W: Wavelengths \# B: Wavebands \#

Fig. 1. An illustration of the PBABL and the MPABWL schemes from node 2 to node 4 because there is no free waveband along route (2-1-4), even though there is a free waveband along route (2-3-4). The constraints on setting up working and backup paths in PBABL are summarized as follows.

(1) For each connection request, the working path is SRLGdiverse from the backup path.

(2) A working waveband-path is set up with a backup waveband-path.

(3) The backup lightpath cannot be shared with other connections.

(4) The backup waveband-path cannot be shared with other connections as a working waveband-path.

(5) The waveband-paths cannot group the connections from different sources or destined to different destinations.

\section{Mixed-Protection-At-waveBand-And-Wavelength-Level $(M P A B W L)$}

MPABWL is similar to PBABL except that it allows a working waveband-path to be protected by several backup lightpaths. For example, in Fig. 1(d), there are two connection requests from node 2 to node 4 . There is a free waveband along route (2-3-4). However, there is no free waveband along route (2-1-4). MPABWL sets up a working waveband-path for the two requests along route (2-3-4) and two backup lightpaths along route (2-1-4). The constraints on setting up working and backup paths for MPABWL are the same as for PBABL except for constraint (2), which limits a working wavebandpath to be protected either by a waveband-path or by multiple lightpaths. In both the schemes, the working paths and the backup paths carry traffic at the same time. When a failure occurs in the working path, the end nodes of the affected lightpaths and waveband-paths just receive and process the traffic in the backup paths.

\section{PBABL HEURISTIC AND MPABWL HeURISTIC}

To obtain the optimum solution, mixed integer linear programming (MILP) can be applied for small networks. For medium and large networks, as the variables and constraints increase exponentially, it is difficult for the MILP approach to find the optimum solution given limited computational resources. Thus, we propose heuristic algorithms adopting random optimization technique for both the schemes. 
DOI: 10.1109/GLOCOM.2005.1577992

Given a connection request, the whole SWBS problem can be solved by adopting three sub-processes in sequence. The first sub-process solves the routing problem which is to obtain a pair of SRLG-diverse paths. The second subprocess assigns available wavelengths/wavebands. Finally, the optimization sub-process adjusts the selected path-pair to maximize the total revenue or to minimize the total provisioning costs. The objective of the cstMIN problem implies that all connection requests can be successfully provisioned, where the provisioning costs are the number of occupied links. Thus, the solution of the revMAX problem with the maximum value can be applied as the initial solution for the cstMIN problem.

The inputs are the network topology and the traffic $\Phi=$ $\left\{\phi_{i}\left(s_{i}, d_{i}, x_{i}, r_{i}, l_{i}\right)\right\}$, where $\phi_{i}$ is the identifier of the group of connection requests, $i$ is the group identifier, $s_{i}$ is the source node, $d_{i}$ is the destination node, $x_{i}$ is the number of connection requests from source $s_{i}$ to destination $d_{i}, r_{i}$ is the revenue set for each connection in the group, and $l_{i}$ is the length constraint set for each connection in the group. Without loss of generality, we let $\phi_{i}\left(x_{i}\right) \leq \theta$. The outputs of the heuristics are the working and backup lightpaths or waveband-paths for the requests.

\section{A. PBABL heuristic for the revMAX problem}

Given connection requests $\phi_{i}$, the whole PBABL heuristic process works as follows.

I. If $\phi_{i}\left(x_{i}\right) \geq 2$, search for the shortest path-pair with enough free wavelengths and wavebands. Assign the first-fit wavelengths/waveband (FFWA/FFWB) to the working path and the last-fit wavelengths/waveband (LFWA/LFWB) to the backup path [7]. If there is no such a path-pair or $\phi_{i}\left(x_{i}\right)=$ 1 , go to Step II. Otherwise, go to Step III.

II. For each connection request from 1 to $\phi_{i}\left(x_{i}\right)$, search for an available shortest path-pair. Adopting FFWA and LFWA for the working and backup lightpaths separately. If there is no such a path-pair, the connection request is blocked.

III. Collect the total revenue of the successfully provisioned connection requests. Adopt the optimization adjustment.

The process first tries to provision the connection requests through a pair of waveband-paths. If the attempt fails, it tries to provision each of the connection requests through a lightpathpair. The optimization process tries to improve the solution. The detailed descriptions of Step I and Step II are shown in Fig. 2 and Fig. 3 respectively. The optimization process will be discussed later in Section III-F.

\section{B. MPABWL heuristic for the revMAX problem}

Given connection requests $\phi_{i}$, the process of the MPABWL heuristic is similar to the one of the PBABL heuristic and is presented as follows.

I. If $\phi_{i}\left(x_{i}\right) \geq 2$, search for the shortest available working waveband-path. If there is no such waveband-path or $\phi_{i}\left(x_{i}\right)=1$, go to Step II. Otherwise, search for an available backup waveband-path. If there is no such a waveband-path, for each connection request in $\phi_{i}$, search for the available shortest backup lightpath. Go to Step III.

\section{Revenue Maximization for the PBABL Heuristic Step I}

1. Obtain a set of up to $k$ shortest working paths for connection requests $\phi_{i}$. Let it be $\chi_{F}=\left\{F_{1}, F_{2}, \ldots, F_{k}\right\}$.

2. If $\chi_{F}=\emptyset$, return with FAIL. Otherwise, continue.

3. For each $F_{i} \in \chi_{F}$.

a) If the number of free wavelengths $w_{F i}<\phi_{i}\left(x_{i}\right)$ along $F_{i}$, eliminate $F_{i}$ and go back to Step 2. Otherwise, continue.

b) If each available waveband along path $F_{i}$ has a capacity of $\theta_{b i}$ and $\theta_{b i}<\phi_{i}\left(x_{i}\right)$, eliminate $F_{i}$ and go back to Step 2. Otherwise, continue.

c) Obtain a set of up to $k$ shortest SRLG-diverse backup paths for $F_{i}$. Let it be $B_{F_{i}}=\left\{B_{i 1}, B_{i 2}, \ldots, B_{i k}\right\}$.

i) If $B_{F_{i}}=\emptyset$, eliminate path $F_{i}$ and go back to Step 2. Otherwise, continue.

ii) For each $B_{i j} \in B_{F_{i}}$,

A) If there are no $\phi_{i}\left(x_{i}\right)$ number of free wavelengths along path $B_{i j}$, eliminate $B_{i j}$ and go back to Step i). Otherwise, continue.

B) If there is no available waveband or the available waveband capacity $\theta_{b}<\phi_{i}\left(x_{i}\right)$ along $B_{i j}$, eliminate $B_{i j}$ and go back to Step i). Otherwise, go to Step d).

d) For the working path $F_{i}$ and the backup path $B_{i j}$, assign wavelengths and wavebands. Return with SUCCESS.

Fig. 2. Description of the PBABL heuristic Step I.

Revenue Maximization for the PBABL Heuristic Step II

For each connection request $\phi_{i}\left(x_{j}\right) \in \phi_{i}$, where $1 \leq x_{j} \leq x_{i}$ :

1. Obtain a set of up to $k$ shortest working paths. Let it be $\chi_{F}=\left\{F_{1}, F_{2}, \ldots, F_{k}\right\}$.

2. If $\chi_{F}=\emptyset$, return with BLOCK. Otherwise, continue.

3. For each $F_{i} \in \chi_{F}$

a) If there is no free wavelength along path $F_{i}$, eliminate $F_{i}$ and go back to Step 2. Otherwise, continue.

b) Obtain a set of up to $k$ shortest SRLG-diverse backup paths for $F_{i}$. Let it be $B_{F_{i}}=\left\{B_{i 1}, B_{i 2}, \ldots, B_{i k}\right\}$.

c) If $B_{F_{i}}=\emptyset$, eliminate path $F_{i}$ and go back to Step 2 . Otherwise, continue.

d) For each $B_{i j} \in B_{F_{i}}$

i) If there is no free wavelength along $B_{i j}$, eliminate $B_{i j}$ and go back to Step c). Otherwise, go to Step e).

e) For the selected working path $F_{i}$ and backup path $B_{i j}$, assign wavelengths.

Fig. 3. Description of the PBABL Heuristic Step II.

II. For each connection request in $\phi_{i}$, find the available shortest path-pair.

III. Collect the total revenue of the successfully provisioned connection requests. Adopt the optimization adjustment.

The process first tries to set up a working waveband-path. If it fails, MPABWL tries to provision the connection requests one by one. The detailed description of Step I is shown in Fig. 4. Step II is the same as the one shown in Fig. 3. The optimization process will be discussed later in Section III-F.

\section{PBABL and MPABWL heuristics for the cstMIN problem}

Both the heuristics take the maximum solutions of the revMAX problem as the initial solutions. They then adopt the optimization process to obtain the optimal or near-optimal solutions, which will be addressed in Section III-F. 
1. Obtain a set of up to $k$ shortest working paths for connection requests $\phi_{i}$. Let it be $\chi_{F}=\left\{F_{1}, F_{2}, \ldots, F_{k}\right\}$.

2. If $\chi_{F}=\emptyset$, return with FAIL. Otherwise, continue.

3. For each $F_{i} \in \chi_{F}$.

a) If $w_{F i}<\phi_{i}\left(x_{i}\right)$ along $F_{i}$, eliminate $F_{i}$ and go back to Step 2. Otherwise, continue.

b) If each available waveband along $F_{i}$ has a capacity of $\theta_{b i}$ and $\theta_{b i}<\phi_{i}\left(x_{i}\right)$, eliminate $F_{i}$ and go back to Step 2. Otherwise, continue.

c) Obtain a set of up to $k$ shortest SRLG-diverse backup paths for $F_{i}$. Let it be $B_{F_{i}}=\left\{B_{i 1}, B_{i 2}, \ldots, B_{i k}\right\}$.

d) If $B_{F_{i}}=\emptyset$, go to Step f). Otherwise, continue.

e) For each $B_{i j} \in B_{F_{i}}$.

i) If $w_{i j}<\phi_{i}\left(x_{i}\right)$ along $B_{i j}$, eliminate $B_{i j}$ and go back to Step d). Otherwise, continue.

ii) If there is no available waveband or the available waveband capacity $\theta_{b}<\phi_{i}\left(x_{i}\right)$ along $B_{i j}$, eliminate $B_{i j}$ and go back to Step d). Otherwise, set up the working waveband-path along $F_{i}$ and the backup waveband-path along $B_{i j}$. Return with SUCCESS.

f) Restore $B_{F_{i}}$, set $F B_{F_{i}}=\emptyset$ and $n W=0$.

g) For each $B_{i j} \in B_{F_{i}}$.

i) Let the number of free wavelengths along $B_{i j}$ be $w_{i j}$. If $w_{i j}>0$, add $B_{i j}$ into $F B_{F_{i}}$ and set $n W+=w_{i j}$.

h) If $n W<\phi_{i}\left(x_{i}\right)$, eliminate $F_{i}$ and go back to Step 2). Otherwise set up the working waveband-route along $F_{i}$ and set up backup lightpaths along the paths in $F B_{F_{i}}$. Return with SUCCESS.

Fig. 4. Description of the MPABWL heuristic Step I.

\section{The Routing Algorithm}

For connection requests $\phi_{i}$, the routing algorithm returns with up to $k$ shortest paths as the candidate working paths. For each candidate working path, it returns with up to $k$ shortest SRLG-diverse paths as the candidate backup paths. We adopt Yen's k-shortest paths algorithm with adjustment for path length constraints [9]. Given a working path $F_{i}$ for $\phi_{i}$, we search for the $k$ shortest SRLG-diverse paths as follows.

1. Eliminate all the links along path $F_{i}$.

2. Eliminate all the links that are not SRLG-diverse from the path $F_{i}$.

3. Using the adjusted Yen's k-shortest paths algorithms to find the loopless and length constrained $k$ shortest paths.

\section{E. The Wavelength and Waveband Assignments}

The objective of the wavelength/waveband assignment is to minimize the total number of utilized wavelength/waveband links. For the working paths, we use FFWA and FFBA algorithms. For the backup paths, we use LFWA and LFBA algorithms [7]. We test other assignment algorithms, which do not show any improvement.

\section{F. The Optimization Process}

The results obtained from the above may not be optimal. The optimization process tries to improve the performance by moving the current solution to another. For a connection request provisioned through lightpaths, the PBABL optimization process tries to obtain a better working lightpath and the

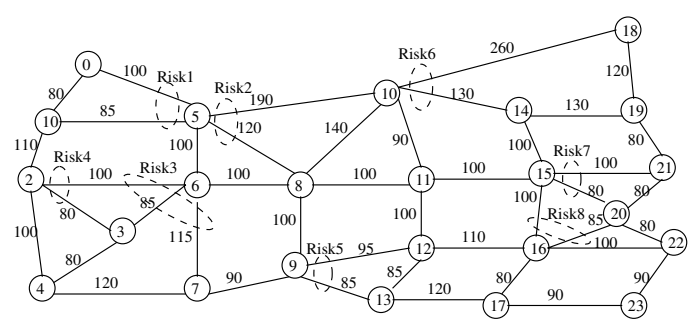

Fig. 5. An example network with 24 nodes and 43 bi-directional links.

MPABWL optimization process tries to obtain a better backup lightpath. We illustrate the optimization process for the cstMIN problem as follows.

The optimization procedure is iteratively adopted to reduce the total number of utilized wavelength links by rearranging the working and backup lightpaths. The waveband-paths will not be rearranged. We randomly pick one connection request in each iteration. In PBABL, if the current selected connection request $\phi_{i}\left(x_{j}\right)$ is provided and protected by waveband-paths, we skip it and proceed to the next connection request $\phi_{\kappa}\left(x_{1}\right)$. Let its working and backup lightpaths be $p_{p}^{\kappa}$ and $p_{b}^{\kappa}$. In MPABWL, if the current selected connection is protected in a waveband-path, we skip it and proceed to $\phi_{\kappa}$ with backup lightpath $p_{b}^{\kappa}$.

The adjustments on working lightpath and backup lightpath are the same except for the wavelength assignments. Thus, we only describe the adjustment on a backup lightpath $p_{b}^{\kappa}$. Let $C_{i j}^{W}$ represent the number of active (used) wavelengths on link $\overrightarrow{i, j}$. Along path $p_{b}^{\kappa}$, the adjustment process searches for the link with the largest value of $C_{i j}^{W}$. It then deletes the link from the network and collects the available backup lightpaths into a set for the connection request. It then searches for the link with the second largest value of $C_{i j}^{W}$ and continues the search in the same manner along $p_{b}^{\kappa}$. Finally, it checks all the lightpaths in the set. Let the checked one be $p_{h}^{\kappa}$. Let $W L_{h}^{\kappa}$ be the number of wavelength-links occupied in $p_{h}^{\kappa}$. If $W L_{h}^{\kappa} \leq W L_{b}^{\kappa}$, replace the backup lightpath $p_{b}^{\kappa}$ by $p_{h}^{\kappa}$. The adjustment stops after running for a predefined number of iterations or until there is no improvement for a predefined number of iterations.

\section{Simulation Results}

We compare results of both the heuristics for both the revMAX and the cstMIN problems. Simulations are conducted on an example mesh network shown in Fig. 5 with 24 nodes and 43 bi-directional links. In Fig. 5, each line represents two unidirectional fibers in opposite directions that are subjected to a common SRLG risk. A dashed circle represents a common SRLG risk and all the links covered by it are subject to the risk. The number on a link represents the link length in kilometers.

The traffic requests $\Phi$ are generated randomly with the length constraints ranging from $900 \mathrm{~km}$ to $1500 \mathrm{~km}$ and with the revenue values ranging from 7.5 to 10.5 . The maximum value of $\phi_{i}\left(x_{i}\right)$ is equal to the waveband granularity $\theta$, which limits the number of connection requests with the same source and destination at the same time. The fiber capacity ranges from 4 to 16 and waveband granularity ranges from 2 to 6 . 
IEEE Global Telecommunications Conference, 2005. GLOBECOM '05. volume: 4

\section{DOI: 10.1109/GLOCOM.2005.1577992}
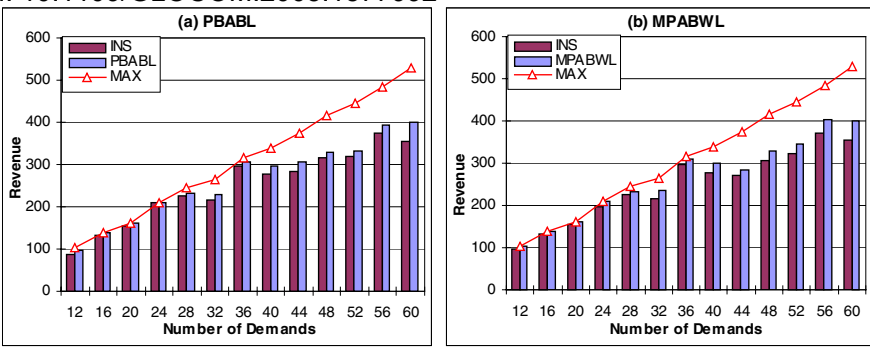

Fig. 6. Results for the revMAX problem. (a) The revenues obtained by the PBABL heuristic compared with the initial solutions. (b) The revenues obtained by the MPABWL heuristic compared with the initial solutions.

The results for the revenue maximization problem are shown in Fig. 6. The lines in the figure show the maximum revenues for the network given the generated connection requests. The value shown by the bar is the average result obtained by running the algorithms 20 times with different fiber capacities and different waveband granularities. Each result is obtained within 1800 seconds for both the heuristics. Fig. 6(a) and Fig. 6(b) show the optimized revenues for PBABL and MPABWL separately compared with their initial results. As can be seen, the initial solutions are almost maximum for 24 or less connection requests. For this reason, the improvements of both the heuristics for the revMAX problem are small under light traffic load. The improvement increases as the network load increases. Fig. 8(a) compares the improved gained revenues of the heuristics. The results are shown in the ratio of the improvement to the maximal revenue. As can be seen, the MPABWL heuristic slightly outperforms the PBABL heuristic in $1 \%$.
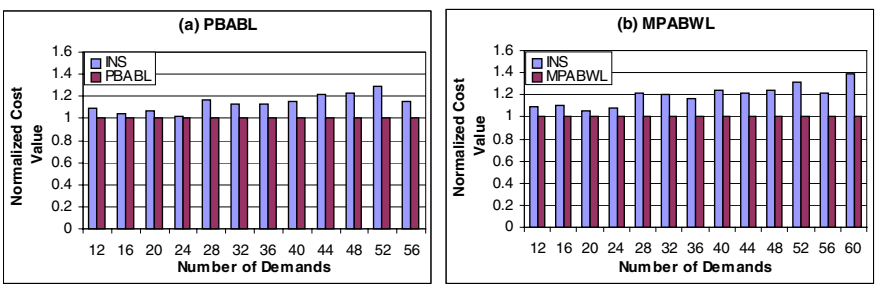

Fig. 7. Results for the cstMIN problem. (a) The number of links utilized in the PBABL heuristic compared with the initial solutions. (b) The number of links utilized in the MPABWL heuristic compared with the initial solutions.
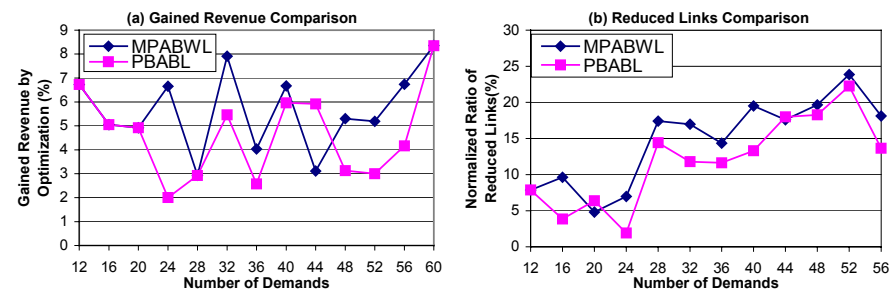

Fig. 8. Comparisons of the performance improvements for the heuristics by adopting optimization process. (a) Comparison of the gained revenue (\%). (b) Comparison of the reduced cost $(\%)$.

The results for the cstMIN problem are shown in Fig. 7. Fig. 7(a) shows the results of the PBABL heuristic and Fig. 7(b) shows the results of the MPABWL heuristic compared with the initial solutions. Each value is the average result obtained by running the heuristics 20 times. The running time is limited to 1800 seconds. To be comparable, the minimum costs obtained by the initial solutions are normalized to the values obtained by the heuristics. The improvements for both the heuristics increase as the number of traffic requests increases. To accommodate the increasing demands, the fiber capacity may also increase. However, our simulation shows that the increase in free wavelengths has little effect on the performance improvement. The improvements of both the heuristics are compared in Fig. 8(b) in terms of the normalized ratio of the reduced cost, which is equal to $1-1 / \operatorname{normal}(I N S)$. The $\operatorname{normal}(I N S)$ represents the normalized value of the initial solution shown in Fig. 7. As can be seen, the normalized ratio of the reduced cost increases as the number of connection requests increases for both the heuristics. We also conclude that for the cstMIN problem, the MPABWL heuristic outperforms the PBABL heuristic by about $2.3 \%$.

\section{CONCLUSIONS}

In this paper, we addressed the survivable waveband switching (SWBS) problem in a WDM mesh network with waveband switching function. Two dedicated path-protection schemes that can accommodate any single point of network failure are proposed, namely the PBABL and the MPABWL schemes. The objectives are to maximize the revenue first and to minimize the costs of provisioned connections next. To deal with the large-sized networks in the real-world, heuristic algorithms utilizing the random optimization technique are proposed. Both heuristics (the PBABL heuristic and the MPABWL heuristic) can obtain very high quality solutions in a short time. Moreover, the results show that the MPABWL heuristic slightly outperforms the PBABL heuristic in most cases.

\section{REFERENCES}

[1] Y. Suemura, I. Nishioka, Y. Maeno, S. Araki, R. Izmailov, and S. Ganguly, "Hierarchical routing in layered ring and mesh optical networks," Proc. IEEE ICC '02, vol. 5, pp. 2727-2733, Apr. 2002.

[2] M. Li and B. Ramamurthy, "Dynamic waveband switching in WDM mesh networks based on a generic auxiliary graph model," Kluwer Journal of Photonic Network Communications, vol. 10, pp. 309-331, Nov. 2005.

[3] A. C. Varsou, S. Ganguly, and R. Izmailov, "Waveband protection mechanisms in hierarchical optical networks," Proc. IEEE HPSR'2003, pp. 317-322, June 2003.

[4] S. Varma and J. P. Jue, "Protection in multi-granular waveband networks," Proc. IEEE GLOBECOM'2004 (Dallas, TX, USA, November-December 2004), vol. 3, pp. 1759-1763, 2004.

[5] D. Zhou and S. Subramaniam, "Survivability in optical networks," IEEE Network, vol. 14, pp. 16-23, Nov.-Dec. 2000.

[6] G. Mohan and C. S. R. Murthy, "Lightpath restoration in WDM optical networks," IEEE Network, vol. 14, pp. 24-32, Nov.-Dec. 2000.

[7] H. Zang, C. Ou, and B. Mukherjee, "Path-protection routing and wavelength assignment RWA in WDM mesh networks under duct-layer constraints," IEEE/ACM Transaction on Networking, vol. 11, pp. 248 258, Apr. 2003.

[8] S. Ramamurthy, L. Sahasrabuddhe, and B. Mukherjee, "Survivable WDM mesh networks," Journal of Lightwave Technology, vol. 21, pp. 870-883, Apr. 2003.

[9] J. Yen, "Finding the k shortest loopless paths in a network," Management Science, vol. 17, pp. 712-716, 1977. 